\title{
When mirrors lie: "Visual capture" of arm position impairs reaching performance
}

\author{
NICHOLAS P. HOLMES, GEMMA CROZIER, and CHARLES SPENCE \\ University of Oxford, Oxford, England
}

\begin{abstract}
If we stand at a mirror's edge, we can see one half of our body reflected in the mirror, as if it were the other half of our body, seen "through" the mirror. We used this mirror illusion to examine the effect of conflicts between visually and proprioceptively specified arm positions on subsequent reaching movements made with the unseen right arm. When participants viewed their static left arm in the mirror (i.e., as if it were their right arm), subsequent right-arm reaching movements were affected significantly more when there was conflict between the apparent visual and the proprioceptively specified right-arm positions than when there was no conflict. This result demonstrates that visual capture of arm position can occur when individual body parts are viewed in the mirror and that this capture has a measurable effect on subsequent reaching movements made with an unseen arm. The result has implications for how the brain represents the body across different sensory modalities.
\end{abstract}

Facing a mirror, we can observe ourselves as we usually see others and as they usually see us - our reflection is leftright reversed (see Gregory, 1996, for an extensive discussion of this phenomenon). Turn the mirror through $90^{\circ}$, however, and now, standing at the mirror's edge, with our head on one side and one arm stretched forward, we can see one side of our body reflected as if it were the other side. Thanks to the almost perfect symmetry of the human body (across the sagittal midline), the visual appearance of one reflected side of our body can provide a strikingly realistic substitute for direct vision of the other side of our body. This mirror illusion has recently been used to great effect in the rehabilitation of neuropsychological patients. For example, mirrors have been used with patients suffering phantom limb pain following arm amputation (e.g., Hunter, Katz, \& Davis, 2003; Ramachandran \& Rogers-Ramachandran, 1996; Ramachandran, Rogers-Ramachandran, \& Cobb, 1995) or with sensorimotor deficits in their arms (Altschuler et al., 1999; Sathian, Greenspan, \& Wolf, 2000).

In addition to the potential clinical uses of this form of mirror therapy, the mirror also offers a powerful experimental tool with which to examine the integration of multiple sources of information concerning the position of our bodies in space, by inducing visual-proprioceptive conflicts (for various examples, see Holmes \& Spence, 2004; Lajoie et al., 1992; Maravita, Spence, \& Driver, 2003). The positions of our hands are specified by a variety of sources of information, including proprioceptive, kinaesthetic, tactile, visual, vestibular, and sometimes even auditory cues

N.P.H. is supported by Wellcome Prize Studentship 065696/Z/01/A from The Wellcome Trust. Correspondence regarding this article should be addressed to N. P. Holmes, Room B121, Department of Experimental Psychology, University of Oxford, Oxford OX1 3UD, England (e-mail: nicholas.holmes@psy.ox.ac.uk). (e.g., think of the sound heard when scratching the back of one's neck!). In most situations, these different cues provide redundant information concerning the true location of our limbs in space. While we are looking in the mirror, however, visual information is often brought into conflict with all the other positional cues. Despite this conflicting visual information, however, we are very good at locating our body parts in the mirror, can readily identify the mirror reflection as that of our own bodies rather than that of someone else behind the mirror, and can, with practice, locate and reach toward external objects while viewing them, using only their mirror reflection to guide us (with certain types of conflict and tasks, however, our performance can be severely impaired; see, e.g., Burnett's detailed studies of "the Aristotle illusion," published in 1904; see also Jackson \& Zangwill, 1953). The ability to perform these apparently simple directed movements in the mirror can, however, be impaired selectively, as the recently discovered neuropsychological syndromes of mirror agnosia and mirror ataxia suggest (Binkofski, Buccino, Dohle, Seitz, \& Freund, 1999; Ramachandran, Altschuler, $\&$ Hillyer, 1997). Conversely, mirror-guided performance in complex visuomotor tasks can actually be enhanced in patients with selective loss of proprioceptive afferent inputs (Lajoie et al., 1992).

In the experiments reported here, we manipulated the position of the right arm held out of sight behind a mirror, while controlling visual cues concerning its apparent position by use of a mirror reflection of the left arm. Participants made unseen reaching movements to a target with the right arm, while looking at the mirror reflection of the left arm or, else, looking at an opaque screen over the mirror. The participants' reaching movements were strongly influenced by the presence of the mirror, suggesting that visual information concerning the apparent position of the right arm can "capture" proprioceptive positional informa- 
tion before (or perhaps during) reaching toward a target. Furthermore, subjective verbal estimates of the initial position of the right hand correlated significantly with the final end-point error of the reaching movements. These results suggest that reaching movements are guided by a combination of visual, proprioceptive, and kinaesthetic cues and that, in the mirror situation, visual cues may play a dominant role over the representation of the initial arm position.

\section{METHOD}

\section{Participants}

Sixteen right-handed participants ( 8 males and 8 females, 20 34 years of age) with normal or corrected vision were recruited by advertisement. The participants gave their informed consent to participate prior to the experiments. All of the participants, apart from 1 (one of the authors, C.S.), were naive as to the purposes of the study. The experiment was approved by the local research ethics committee.

\section{Apparatus and Materials}

A mirror measuring $45 \times 30 \mathrm{~cm}$ was positioned vertically in the middle of a large table, with the reflective surface facing to the participant's left and oriented parallel to the participant's sagittal axis (see Figure 1A). An opaque flat platform measuring $45 \times 45 \mathrm{~cm}$ and raised $20 \mathrm{~cm}$ above the table surface was positioned immediately to the right of the mirror. A small mark was positioned on the table $12 \mathrm{~cm}$ to the left side of the mirror and $30 \mathrm{~cm}$ in front of the participant. Similar marks were positioned 5, 12, 19, and $26 \mathrm{~cm}$ to the right of the mirror, out of the participant's view. A fixed target indicator $(10 \times$ $10 \mathrm{~cm}$ piece of cardboard with a vertical arrow in the middle) target indicator was positioned within the participant's view on top and at the rear of the opaque platform, $12 \mathrm{~cm}$ to the right of the mirror. The target indicator pointed toward a position $12 \mathrm{~cm}$ to the right and at the back edge of the mirror (i.e., $45 \mathrm{~cm}$ in front of the participant). In Experiment 2, a row of printed roman letters (A to $Z$, spaced $1 \mathrm{~cm}$ apart and spanning a total of $25 \mathrm{~cm}$ ) was positioned on top of the occluding platform, starting $3 \mathrm{~cm}$ to the right of the mirror and $30 \mathrm{~cm}$ from the participant's body, running parallel to and directly above the right-arm position marks. An opaque cloth was draped over the participant's right arm and shoulder to minimize any visual cues pertaining to the position of the right arm.

\section{Design}

In Experiment 1, there were two experimental conditions - namely, the mirror condition and the no-mirror condition. The starting condition was counterbalanced across participants. In both conditions, the index finger of the participant's right hand was positioned 5, 12, 19 , or $26 \mathrm{~cm}$ to the right of the mirror. The index finger of the left hand was always positioned $12 \mathrm{~cm}$ to the left of the mirror. Five trials were performed for each of the four right-hand positions and two experimental conditions (i.e., a total of 40 trials per participant). The order of arm positions within each condition was randomized on a trial-by-trial basis. In Experiment 2, an extra condition-namely, mirror-passive - was included. Six counterbalanced block orders were constructed, to which the participants were assigned serially. The participants in Experiment 2 performed 10 trials per hand position and condition (i.e., 120 trials in total) to provide extra data points for a correlation analysis. Ten participants performed Experiment 1, and 6 new participants performed Experiment 2.

\section{Procedure}

The participant sat facing the table and positioned himself or herself just to the left of the mirror. The index finger of the participant's left hand was placed $12 \mathrm{~cm}$ to the left of the mirror and $30 \mathrm{~cm}$ in front of his or her body. The participant's right arm was positioned under the platform, with the right index finger on one of the four marks. The participant was instructed to look at the reflection of his/her left hand in the mirror (specifically, at his/her left index finger). When both left and right hands were positioned on the 12-cm marks, the mirror reflection of the left arm thus appeared to be in a position identical to the true position of the right arm (as if the right arm were seen "through" the mirror). In all other right-hand positions, the visually specified apparent position of the right hand and the proprioceptively specified true position of the right hand conflicted.

In a short practice session at the start of the experiment, the mirror was covered so that the left arm was visible only directly and not in the mirror. The participant practiced reaching with the unseen right index finger to the target position on the table $45 \mathrm{~cm}$ in front of him or her. Feedback on reaching accuracy was given by the experimenter, and practice continued until the participant could reach consistently with the index finger, landing within $2 \mathrm{~cm}$ of the target point. No further feedback was given after this practice session.

The mirror was then uncovered, and the participant was asked to move the fingers of the left and right hands synchronously and in an identical manner, until he or she felt that the mirror reflection of the left hand seemed to be identical to the real right hand. This latchingon to the mirror reflection was a purely subjective judgment and, in the practice session, served only to illustrate to the participant the nature of the mirror illusion he or she was expected to experience. In the experimental sessions, this latching-on was the crucial determinant of the timing of the subsequent reaching movement, as will be described in more detail below. The participant was always aware of the presence or absence of the mirror and the identity of the hand (i.e., the left) seen reflected in it, although the onset and existence of the mirror illusion itself suggests that, in some sense, the participant was unsure of which hand he or she was viewing in the mirror. (This uncertainty is made particularly vivid when, after experiencing the illusion, one hand is moved while the other remains still.)

Each experimental trial began with the experimenter moving the index finger of the participant's right hand under the screen into a position $5,12,19$, or $26 \mathrm{~cm}$ to the right of the mirror. When the hand was in position, the participant was asked to begin tapping the fingers of the left and right hands synchronously and in an identical manner until he or she experienced the onset of the mirror illusion, which was reported verbally to the experimenter. In the no-mirror condition, the participant was instructed to move the fingers for approximately $6-8 \mathrm{sec}$ (the average illusion onset time as determined by earlier pilot research). Several seconds after the reported onset of the illusion (or after about $10 \mathrm{sec}$ in the no-mirror condition), the experimenter gave the instruction to "reach," at which point the participant gazed toward the target indicator and reached forward with his/her right arm and attempted to place the right index finger on the target position directly below the indicator. The participant was instructed to make one quick, smooth movement with his/her right arm and to reach to the target as accurately as possible, leaving the index finger touching the table until the experimenter had recorded the reaching error (in centimeters to the left or right of the target with respect to the participant) with a ruler. The participant's arm was then set to a new position, and the next trial began.

The no-mirror condition differed from the mirror condition in that an opaque covering was placed over the mirror and the participant was instructed to look at the covering as if he or she were looking through it toward the unseen right index finger (when placed in the $12-\mathrm{cm}$ position). This same gaze direction was adopted for all the right-arm positions.

Experiment 2 was identical to Experiment 1, except for the following differences. Immediately prior to reaching in the mirror and nomirror conditions, the participant was instructed to look at a line of printed letters on top of the occluding platform and to report verbally the letter that he or she felt was directly above the tip of the right index finger. When he or she had reported a single letter, he or she reached toward the target as in Experiment 1. The participant was en- 
A

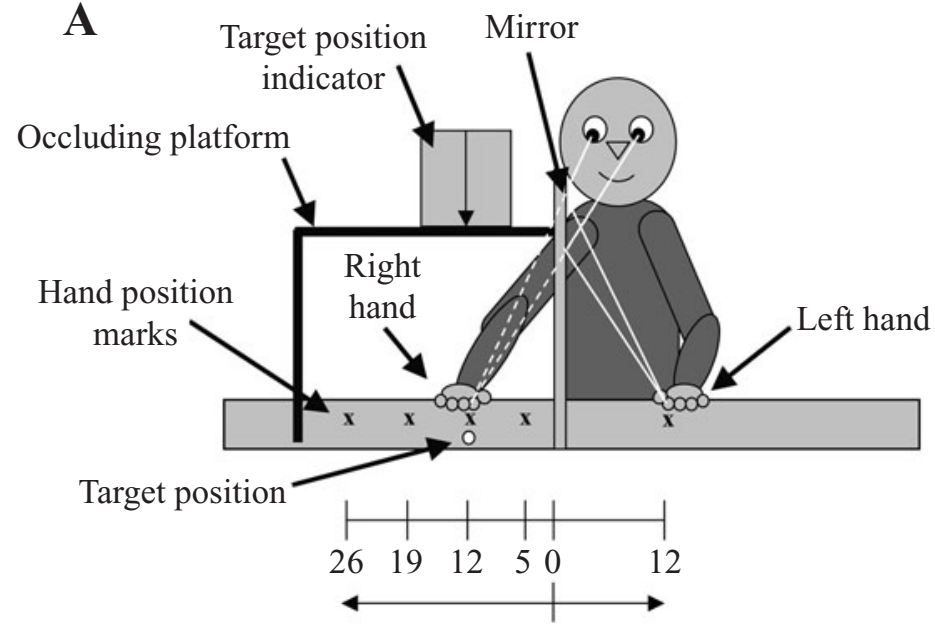

Right-hand position (cm right of mirror)

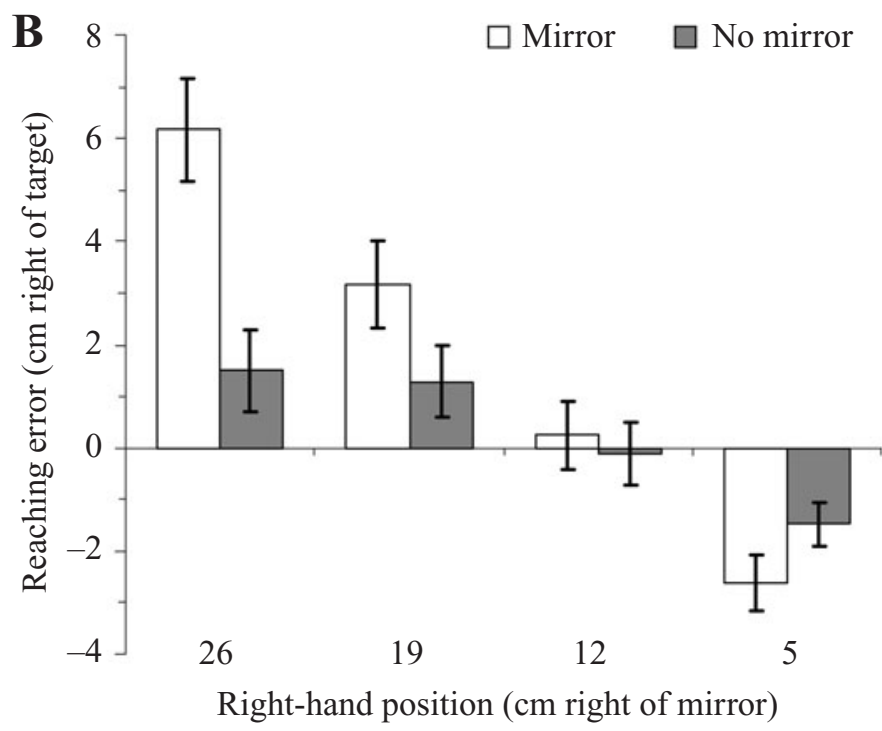

Figure 1. Experimental apparatus and results of Experiment 1. (A) The participant sat at a table, with a mirror positioned vertically in the center, and an occluding platform to the participant's right. The participant's right hand was placed by the experimenter on one of the four hand positions marked on the table. The right arm and hand were never visible to the participant during the experiment, whereas the left arm and hand were visible directly in both conditions and via the mirror reflection in the mirror condition. When the right hand was positioned $12 \mathrm{~cm}$ to the right of the mirror, the mirror reflection of the left hand appeared to be in the same position as the true location of the right hand (as is shown in the figure; solid and broken white lines show the approximate line of sight, via the mirror reflection, of the left hand). In all other right-hand positions $(5,19$, and $26 \mathrm{~cm})$, the apparent visual appearance of the right hand (really the mirror-reflected left hand) was mismatched with respect to the true position of the right hand. After tapping the fingers of both hands synchronously and experiencing the mirror illusion (the feeling that one can see through the mirror to observe one's right hand directly), the participant reached with his or her right index finger to the position on the table where he or she judged the target position indicator line would meet the table (i.e., $12 \mathrm{~cm}$ to the right of the mirror and $45 \mathrm{~cm}$ in front of the participant). (B) The bars show the mean ( \pm standard error) of mean reaching errors across participants, separately for both conditions and the four hand positions. Open bars, mirror condition; filled bars, no-mirror condition; $x$-axis, distance of right index finger from the mirror before reaching; $y$-axis, mean reaching error (measured in centimeters to the right of the target). 
couraged to be as accurate as possible in reporting the felt location of the right index finger but, also, not to take more than 1 or $2 \mathrm{sec}$ to do so. Verbally reported illusion onset times were not measured in Experiment 2. The participant was instructed to report the letter, then reach toward the target when he or she began to feel the mirror illusion in the mirror condition (rather than when instructed to do so, as in Experiment 1) and after approximately $6 \mathrm{sec}$ of finger-tapping in the no-mirror condition. In addition, in the mirror-passive condition of Experiment 2, the participant was asked simply to fixate on the mirror-reflected left index finger for approximately $2 \mathrm{sec}$ before reaching directly to the target as described above. This extra condition served to examine the effect of a brief visual exposure to the mirror situation in the absence both of movements of the two hands and of a conscious effort to experience the mirror illusion.

\section{Data Analysis}

The measure of interest was the reaching error of the index finger of the right hand. The landing point of the right index finger was recorded in one dimension only- that is, the distance between the target and the landing point measured perpendicular to the mirror. Negative values indicate a landing point too close to the mirror, whereas positive values indicate a landing point too far from the mirror. In all cases, the target was positioned $12 \mathrm{~cm}$ to the right of the mirror; thus, all measurements were taken from this origin. In addition, for each trial in Experiment 1, the time taken to experience the illusion, according to the participant's subjective report, was recorded, and for each trial of the mirror and no-mirror conditions in Experiment 2 , the letter reported by the participant was recorded.

Error data were analyzed using a two-way analysis of variance (ANOVA) with the factors of condition (mirror vs. no mirror) and right-hand position $(5,12,19$, or $26 \mathrm{~cm})$. Post hoc tests were performed to examine individual levels of the main experimental factors. Illusion onset times were analyzed with a one-way ANOVA. The hand position judgment data from Experiment 2 were analyzed on a trial-by-trial basis with the reaching error separately for each participant, using Pearson's product-moment correlations. The $r$ values and slope coefficients of the regression equations for these correlations were compared between conditions, using Student's one-tailed paired $t$ tests. All measurement data given are the means of individual means, plus or minus the standard errors of the means (SEMs).

\section{RESULTS}

\section{Experiment 1}

The mean reaching error data averaged across participants for both the mirror and the no-mirror conditions and for each hand position are presented in Figure 1B. The analysis of the reaching error revealed a significant main effect of condition $[F(1,9)=28.46, p<.001]$. Comparison of the mean reaching error for the mirror $(1.74 \pm 0.69 \mathrm{~cm})$ versus the no-mirror $(0.3 \pm 0.59 \mathrm{~cm})$ conditions revealed a significantly larger reaching error in the mirror condition (mean \pm SEM difference between conditions $=1.44 \pm$ $0.27, p<.001)$. Since the reaching error was always negative when the right hand was in the $5-\mathrm{cm}$ position, the main effect of condition in this analysis is underestimated. If the reaching errors were, instead, converted to absolute errors (i.e., in terms of their distance from the target regardless of direction), the mean $\pm S E M$ of the differences between the conditions was $1.67 \pm 0.35 \mathrm{~cm}$.

The main effect of hand position was also significant $[F(3,27)=62.01, p<.001]$. Post hoc Bonferroni tests between individual hand positions revealed statistically reli- able differences between all comparisons (all mean differences $>1.6 \mathrm{~cm}$; all $p \mathrm{~s}<.01$ ). The significant trend in these data is toward larger reaching errors with increasing separation of the right hand from the target line. Furthermore, in all cases, the mean reaching error shows an underestimation of the distance to the target (positive values for the 19- and $26-\mathrm{cm}$ hand positions, negative values for the $5-\mathrm{cm}$ hand position).

The crucial experimental comparison concerns the interaction between the mirror condition and hand position. If the visual appearance of the mirror reflection of the left hand affected the felt position of the right hand (i.e., if it visually captured the felt position of the right hand), pointing errors should have increased as the discrepancy between the visually and the proprioceptively specified hand positions increased, and this increase should have been larger for the mirror condition than for the no-mirror condition. In short, the interaction between condition and hand position should have been significant. The ANOVA revealed this to be the case $[F(3,27)=44.5, p<.001]$. Post hoc tests between the mirror and the no-mirror conditions for individual hand positions revealed that the differences between mean reaching errors when the right hand was placed $5 \mathrm{~cm}[t(9)=-2.94, p<.05], 19 \mathrm{~cm}[t(9)=$ $4.94, p<.01]$, and $26 \mathrm{~cm}[t(9)=8.34, p<.001]$ from the mirror were all significant, whereas there was no significant difference between conditions when the right hand was placed $12 \mathrm{~cm}$ from the mirror.

The mean reaching error across participants was significantly correlated with hand position in both the mirror condition $(r=1, p<.005)$, and the no-mirror condition $(r=.96, p<.005)$. However, in the mirror condition, the slope of the best-fit line through the means was 0.42 , as compared with a slope of only 0.15 in the no-mirror condition. Thus, for every centimeter that the right hand was moved away from the target in either direction, the reaching error increased in the same direction by an average of $0.42 \mathrm{~cm}$ in the mirror condition and by only $0.15 \mathrm{~cm}$ in the no-mirror condition.

The analysis of the time taken to experience the illusion revealed no main effect of hand position, although the difference between the 26 - and the $19-\mathrm{cm}$ positions was significant after correcting for multiple comparisons $(26 \mathrm{~cm}$, $8.1 \pm 0.8 \mathrm{sec} ; 19 \mathrm{~cm}, 6.2 \pm 0.6 \mathrm{sec} ; p<.05$, one-tailed $t$ test). For comparison, mean illusion times for 5 - and $12-\mathrm{cm}$ hand positions were $6.2 \pm 1.0 \mathrm{sec}$ and $6.1 \pm 0.6 \mathrm{sec}$, respectively.

\section{Experiment 2}

The results of Experiment 2 were very similar to those of Experiment 1. Analysis of the reaching error for the 6 additional participants revealed a significant main effect of position $[F(3,15)=24.01, p<.001]$ and a significant interaction between condition and position $[F(6,30)=14.05$, $p<.001]$. There was no significant main effect of condition. Further analysis of the condition $\times$ position interaction revealed significant differences between all position pairs (planned pairwise comparisons) for the mirror and 
mirror-passive conditions (all $F_{\mathrm{s}}>6.3$, all $p \mathrm{~s}<.05$ ) and the mirror and no-mirror conditions, but no significant differences between the mirror-passive and the no-mirror conditions (all $F_{\mathrm{s}}<1, n$.s.). The best-fit regression lines through the means of the reaching errors had slope coefficients of 0.30 (mirror condition), 0.08 (no-mirror condition), and 0.10 (mirror-passive condition).

The index finger position judgments made in the mirror condition were significantly correlated with reaching error measurements on a trial-by-trial basis. Individual participants' $r$ values for the mirror condition ranged from .35 to .87 (all $p \mathrm{~s}<.025$ ), whereas in the no-mirror condition, the correlations between these two measures were much lower (range, -.11 to $.53 ; 4$ participants, $p<.05$; 2 participants, $p>.05$ ). Figure 2 shows the relationship between initial finger position judgment and final reaching error for 2 participants. Across all 6 participants, the coefficients of the best-fit regression slope for the mirror $\left(0.54 \pm 0.17 \mathrm{~cm}^{-1}\right)$ and the no-mirror $\left(0.07 \pm 0.03 \mathrm{~cm}^{-1}\right)$ conditions were significantly different from each other $[t(5)=4.05, p<.01]$, indicating that every centimeter difference in reported initial finger position had a greater influence on reaching error in the mirror condition (i.e., by $0.54 \mathrm{~cm})$ than in the no-mirror condition $(0.07 \mathrm{~cm})$. The point for each participant at which the regression slopes for the mirror and the no-mirror conditions crossed (i.e., the perceived initial hand position related to the same average reaching error in both the mirror and the no-mirror conditions) was $10.2 \pm 2.2 \mathrm{~cm}$ to the right of the mirror (mean and $S E M$ of crossing points for 6 participants, n.s.d. from the actual point of visual-proprioceptive equality, at $12 \mathrm{~cm}$ to the right of the mirror).

\section{DISCUSSION}

When the participants reached toward a target with their unseen right hands, the presence of a mirror reflection of their left hands significantly influenced the accuracy of their reaching (at least when tested immediately after experiencing the mirror illusion). The influence of the mirror reflection was strongest when the discrepancy between the apparent initial position of the right hand (visually specified) and the real initial position of the right hand (proprioceptively specified) was largest. The reaching error increased as a function of the absolute separation of hand and target position in both the mirror and the no-mirror conditions, but this increase was much greater in the mirror condition (slope $=0.42$ in Experiment 1, 0.30 in Experiment 2) than in the no-mirror condition (slope $=0.15$ in Experiment 1, 0.08 in Experiment 2). Thus, the present experiments demonstrate that the mirror illusion, where the reflected left hand both appears to occupy the space of

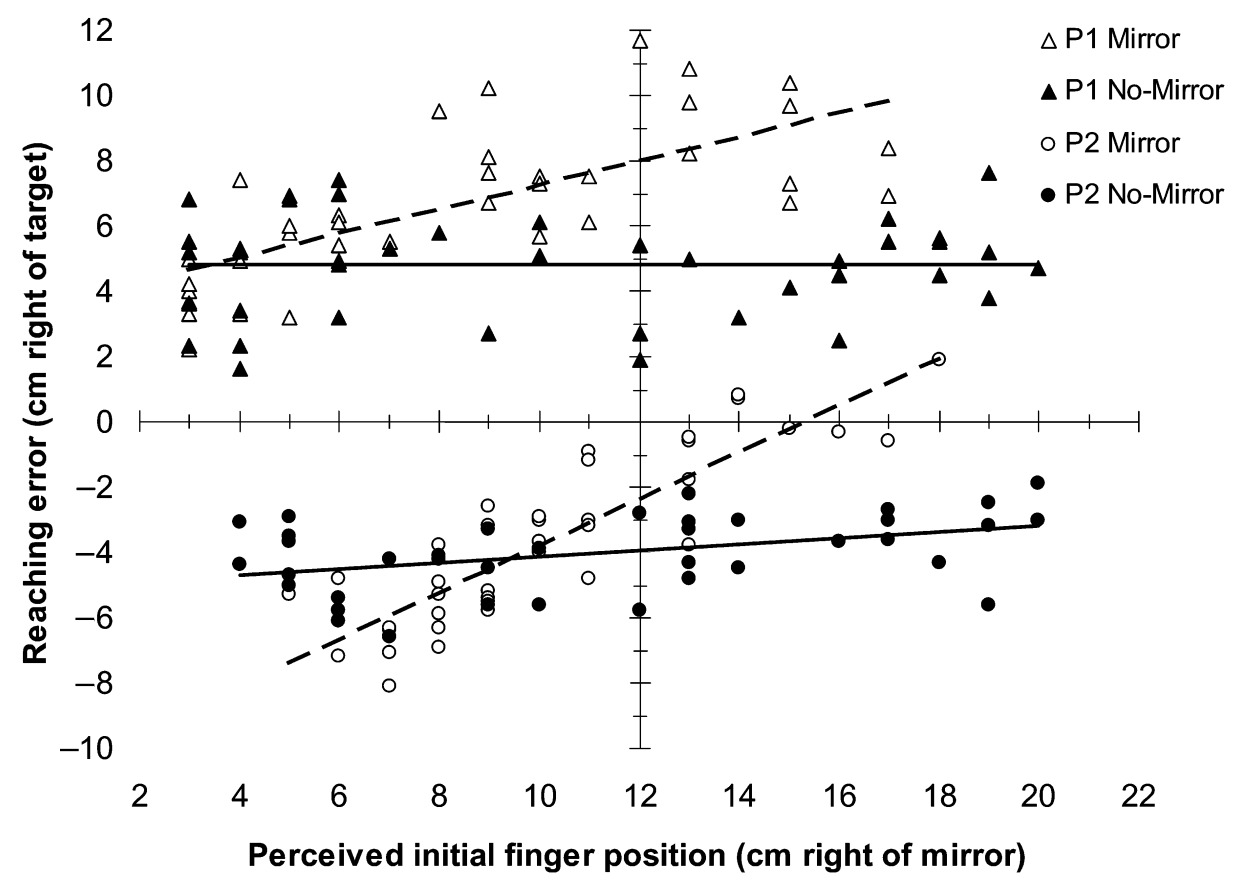

Figure 2. Scatterplots of the reaching error ( $y$-axis) against the reported initial finger position ( $x$ axis), for 2 representative participants in Experiment 2 (selected for clarity of display). Each data point represents one trial. Perfect performance would be evidenced by a horizontal line of data points along the $x$-axis (i.e., with a reaching error of zero). Conversely, complete visual capture at all initial right-hand positions would be evidenced by a vertical line of data points running along the $y$-axis (the participant would always feel his or her hand to be $12 \mathrm{~cm}$ right of the mirror). Triangles, Participant 1; circles, Participant 2; open symbols and broken regression lines, mirror conditions; filled symbols and solid regression lines, no-mirror conditions. 
the right hand and, moreover, actually feels as if it is the right hand, can have a measurable effect on subsequent unseen reaching movements made with the right hand. Furthermore, as was shown by Experiment 2, the perceived initial position of the unseen finger was highly correlated with the final reaching error, suggesting that the effect of the felt position of the right hand was crucial in producing the reaching errors we observed. This conclusion is made even stronger by the results of the mirrorpassive condition in Experiment 2: When the participants made reaching movements after just $2 \mathrm{sec}$ of passive visual exposure to the reflected left hand (as compared with an average of approximately $6 \mathrm{sec}$ active experience in the mirror condition), there was little modulation of reaching performance, as compared with the no-mirror condition. The slight difference in the regression slope between the mirror-passive $(0.10)$ and the no-mirror $(0.08)$ conditions may be suggestive of a residual effect of visual information alone, which should be addressed in future studies. It would be interesting to study the effect of visual exposure time on any modulation of reaching error in both active and passive mirror conditions: Can the same level of reaching error be produced with active and passive conditions, and is there a minimum exposure time for any effects on reaching error to occur?

\section{The Source of the Reaching Errors}

What might account for the effect of the mirror reflection on the participants' reaching performance? The simplest explanation, that the participants were unaware that they were not viewing their right hands, can be discounted. All of the participants were aware of the mirror's presence at all times, and at no point could they see their right hands or arms. Indeed, as soon as the participant made a reaching movement with the right hand, the lack of any visual feedback regarding this movement would have been registered. A second explanation might be that participants were simply biased by the experimental situation toward making errors in a certain direction. This can presumably be discounted, since the experimenter encouraged the participants always to reach as accurately as possible to the target, the indicator for which was always in view. An explanation based on a constant reaching error (which differed between conditions) can also be discounted, since the direction of the reaching error reversed when the right hand was brought closer to the mirror (at the $5-\mathrm{cm}$ position) and the correlation of hand position with mean reaching error was perfect $\left(r^{2}=1\right.$ in Experiment 1, 0.998 in Experiment 2). Finally, an explanation in terms of proprioceptive drift throughout the pointing trials (which were performed under open loop conditions without visual feedback or information about reaching accuracy) can be discounted, since the trial presentation order was randomized, so any gradual proprioceptive drift would have affected all right-hand positions equally. We believe the most appropriate explanation to involve the effect of conflicting visual information concerning the (apparent) position of the right hand on subsequent reaching movements made with that hand. This effect is a form of visual capture of initial hand position in the mirror. One question that remains to be determined is to what extent the effects observed here are due simply to the displaced visual image of the hand or to the feeling that the hand is one's own. Initial results from our second experiment suggest that it is largely the felt position of the hand that determines the direction and a large proportion of the magnitude of the reaching error, rather than simply the visual image of the hand.

That the effect of the mirror illusion was not complete (reaching error was an average of $30 \%-42 \%$ of the difference between initial left- and right-hand positions in the two experiments) might be explained in several ways. First, insufficient time may have elapsed for the full illusion to take hold. Longer experience of the sensory conflict might lead to greater recalibration of reaching performance, along with an enhanced feeling of the mirror illusion, although it is an open question as to whether visual capture occurs prior to any visual-proprioceptive recalibration (which leads to the reaching errors) or whether recalibration is a necessary precondition for visual capture to occur. Second, the visual modality was only one source of limb position information. All the other cues combined (proprioceptive, somatosensory, and auditory cues, plus any "higher," cognitive influences-e.g., remembering in which direction one's hand was moved at the beginning of a given trial and deliberately compensating for this directional shift when reaching) might also have limited the effect of the conflicting visual information concerning hand position. Finally, once the reaching movement began, dynamic positional or kinaesthetic information was also available to the participant, and mid-reach trajectory corrections may have occurred (although see Rossetti, Desmurget, \& Prablanc, 1995). Examining the trajectory of the right hand during the reaching movement would allow the effect of this dynamic position information to be assessed in more detail.

\section{Visual Capture by Different \\ Experimental Means}

In a study with experiments similar to the experiments reported here, Rossetti et al. (1995) asked participants to reach with their right hands toward one of four visible virtual targets in a darkened room from one initial starting position. Prior to the reaching movement, the participants received a brief visual cue (the illumination of a yellow LED attached to the index fingertip) concerning the position of the reaching hand, but throughout the reaching movement, only the target was visible, and no positional feedback was given concerning hand position. In one condition, the initial hand position was displaced $8.5^{\circ}$ to the right by lenses mounted in the mirror used to display the virtual targets, whereas in the control condition there was no visual displacement of initial hand position. In both conditions, vision of the targets themselves remained undistorted throughout the experiment. Rossetti et al. showed that in the displaced hand condition, reaching movements were biased by a constant amount (approximately $13 \mathrm{~mm}$, or $32 \%$ of the total visual displacement) toward the visually specified initial hand position for all four targets. Since no vi- 
sual feedback of the hand was given during the reaching movements, this result suggests that adaptation to conflicts between visually and proprioceptively specified hand position can be as large as $30 \%$ of the disparity simply on the basis of initial hand position alone. Our result of a $30 \%-$ $42 \%$ bias toward vision (or more specifically, a $22 \%-27 \%$ visually induced proprioceptive bias in the mirror condition, added to the $8 \%-15 \%$ residual reaching bias in the no-mirror and mirror-passive conditions), along with the lack of visual feedback concerning the reaching movement itself, therefore accords well with Rossetti et al.'s findings.

In a study of the effects of adaptation to prism-induced shifts of the visual field on the ability to match the position of left and right index fingers, Mon-Williams, Wann, Jenkinson, and Rushton (1997) found that matching errors were lower for an active matching task than for a more passive matching task. When participants moved their visible right hands to a target and then moved their unseen left hands to match the position of the right hands under a table, the matching error was $63 \%$ of the visual matching error (which was fully biased by the prismatic shift). When the right hands were placed on the target by the experimenter (passive condition), the matching error was now biased $90 \%$ toward the visual displacement. The finding of an increased ability to localize body parts when moved actively in Mon-Williams and colleagues' study contrasts with the decreased reaching accuracy we found when participants moved their fingers actively prior to reaching. This dissociation of the effects of active and passive movements on visual capture and sensorimotor adaptation to prismatic displacement suggests that these two processes may depend on quite different neural systems (see Holmes \& Spence, 2004, for a discussion of a variety of methodological approaches to these issues; see also Welch, Widawski, Harrington, \& Warren, 1979).

Our findings raise important theoretical questions concerning the relationship between visual capture induced by different means. Hay, Pick, and Ikeda (1963) demonstrated visual capture of felt arm position when participants wore displacing prisms over their eyes, and there are more recent examples of this phenomenon (e.g., MonWilliams et al., 1997). Another form of visual capture is that induced by artificial body parts. This phenomenon was first studied by Tastevin in 1937, who reported that observers tended to attribute proprioceptive sensations to a visible body part (an artificial finger) when their own corresponding body part was not visible. This visual "capturing" of proprioception by artificial body parts has been demonstrated more recently by Botvinick and Cohen (1998), who replicated Tastevin's findings solely on the basis of subjective report and demonstrated a significant empirical effect of visual capture on reaching performance. Pavani, Spence, and Driver (2000) have studied this phenomenon, using a visuotactile interference paradigm. They examined the effect of visual distractors on tactile discrimination reaction times when the distractors were presented near a rubber arm that was either in an anatomically realistic alignment with the participant's own body or in an unrealistic position. When the rubber arm was in alignment with the participant's body, the effect of the visual distractors was significantly stronger than when the arms were not aligned, suggesting that an artificial, but plausibly aligned, arm can visually capture tactile stimuli. These recent methodological approaches could be integrated into the present mirror experiments: What, for example, would be the effect of seeing a rubber arm in the mirror in place of one's own arm? If there is some visual capture with a rubber arm, what is the effect of a misaligned, alien, or asymmetric rubber arm on this phenomenon (see Austen, Soto-Faraco, Eich, Enns, \& Kingstone, 2003; Austen, Soto-Faraco, Pinel, \& Kingstone, 2001; Pavani et al., 2000; and see Niebauer, Aselage, \& Schutte, 2002, and Peled, Ritsner, Hirschmann, Geva, \& Modai, 2000, for participant-specific influences on the rubber hand illusion)?

The present experiments present a new kind of visual capture induced by a mirror (see also Nielsen, 1963, and Ro, Wallace, Hagedorn, Farnè, \& Pienkos, 2004, for related findings). This method has several benefits over the prismatic displacement or artificial body part techniques used in many previous studies. First, during prismatic displacement, the whole visual field is usually shifted by an equal amount in one direction, which does not allow a body part specific manipulation of visual and proprioceptive information. Visual capture due to prism adaptation could, therefore, affect all subsequent pointing movements, not just those concerned with individual body parts, although the method of Rossetti et al. (1995) overcomes this problem to some extent. Moreover, several different neural mechanisms may be implicated in prism adaptation, such as those involved in the control of eye movements and hand-eye coordination, as well as those concerned with representing the position of individual body parts or representing allocentric visual space (see Harris, 1965, for a review of various sensorimotor consequences of displaced vision). Second, the use of a mirror to present visual information regarding limb position allows for the possibility of studying active movements made out of sight while a passive limb is still always visible. The use of mirrors, prisms, and artificial body parts to investigate the different sensorimotor mechanisms contributing to the visual capture of somatosensory and proprioceptive information may best be used in conjunction with similar tasks (see Holmes \& Spence, 2004; Maravita et al., 2003). It might be, for instance, that these three experimental techniques affect independent sensorimotor systems and are, thus, not directly comparable. Alternatively, they may all influence the same sensorimotor system or systems, and the findings of one task may be replicated exactly with a different methodology. The truth probably lies somewhere between these extremes, and the different manipulations presumably make different demands on different, but closely interacting, sensorimotor systems.

\section{Neural Substrates of Rubber-Arm and Mirror Illusions}

What might be the neural correlates of the behavioral effects we have found here? Several interesting properties of single cells in the posterior parietal cortex of macaque 
monkeys may be of relevance to these behavioral effects. For example, Graziano, Cooke, and Taylor (2000) recorded from single cells in the posterior parietal cortex (Brodmann's area 5) of macaque monkeys, which seemed to represent specific arm positions. Single neurons in this cortical area were shown to fire more strongly, for example, when the monkey's left arm was positioned on the left side than when it was positioned on the right side. Some of these cells would also increase their response when a taxidermied left monkey arm was presented on the left side, but not to a stuffed right arm presented on the left side - that is, these cells were sensitive to the apparent mirror reversal of the arm. It is tempting to speculate that cells in the human posterior parietal cortex may also be responsible for mediating the behavioral effects of the mirror illusion reported here. When presented with a visual image of the (apparent) right arm, in a position on the right side of space, such cells would increase their firing rates accordingly, signaling the apparent position of the right arm. Other cells, which are not sensitive to the discrepant visual information (approximately half of all cells in the study by Graziano et al., 2000), would signal the proprioceptively specified position of the right arm. If the two sources of information were congruent, subsequent reaching accuracy would be unaffected. If there was an inconsistency, however, reaching movements might be biased according to the strength of the two signals and according to other signals arising before or during the subsequent reaching movement.

\section{Summary and Conclusions}

The results of the present study illustrate the dominance of visual information over other sources of positional information when we view our body in a mirror. One consequence of this visual dominance is that participants subjectively reported that the mirror-reflected left hand, when actively moved in mirror symmetry and in synchrony with the unseen right hand, felt like it was the right hand. Another consequence, closely correlated with the subjective impression of hand position, is that reaching movements to an unseen target were significantly affected by the mirror illusion. It seems that, even though we may "know" that our hand is in one position, we cannot help but be influenced by the apparent sight of it in another position. This plasticity of the brain's multisensory representation of the body in space may explain the remarkable capacity of humans and some other primates to guide their own movements and even to recognize themselves in the mirror.

\section{REFERENCES}

Altschuler, E. L., Wisdom, S. B., Stone, L., Foster, C., Galasko, D., Llewellyn, D. M., \& Ramachandran, V. S. (1999). Rehabilitation of hemiparesis after stroke with a mirror. Lancet, 353, 2035-2036. Austen, E. L., Soto-Faraco, S., Eich, E., EnNs, J. T., \& Kingstone, A. F. (2003). Limits to body schema remapping in the fake hand illusion. Abstracts of the Psychonomic Society, 8, 112.

Austen, E. L., Soto-Faraco, S., Pinel, J. P. J., \& Kingstone, A. F. (2001). Virtual body effect: Factors influencing visual-tactile integration. Abstracts of the Psychonomic Society, $6,2$.

Binkofski, F., Buccino, G., Dohle, C., Seitz, R. J., \& Freund, H.
(1999). Mirror agnosia and mirror ataxia constitute different parietal lobe disorders. Annals of Neurology, 46, 51-61.

Botvinick, M., \& Cohen, J. (1998). Rubber hands "feel" touch that eyes see. Nature, 391, 756 .

BurnetT, C. T. (1904). Studies in the influence of abnormal position upon the motor impulse. Psychological Review, 11, 370-394.

Graziano, M. S. A., Cooke, D. F., \& TaYlor, C. S. R. (2000). Coding the location of the arm by sight. Science, 290, 1782-1786.

Gregory, R. L. (1996). Mirrors in mind. New York: Freeman.

HARRIS, C. S. (1965). Perceptual adaptation to inverted, reversed and displaced vision. Psychological Review, 72, 419-444.

HAY, J. C., PICK, H. L., JR., \& IKEDA, K. (1963). Visual capture produced by prism spectacles. Psychonomic Science, 2, 215-216.

Holmes, N. P., \& Spence, C. (2004). Body schema and body image: Toward a taxonomy of multisensory bodily manipulations. Manuscript submitted for publication.

Hunter, J. P., Katz, J., \& Davis, K. D. (2003). The effect of tactile and visual sensory inputs on phantom limb awareness. Brain, 126, 579-589.

JACKsON, C. V., \& ZANGWILl, O. L. (1953). Experimental finger dyspraxia. Quarterly Journal of Experimental Psychology, 4, 1-10.

Lajoie, Y., Paillard, J., Teasdale, N., Bard, C., Fleury, M., ForGET, R., \& LAMARRE, Y. (1992). Mirror drawing in a deafferented patient and normal subjects: Visuoproprioceptive conflict. Neurology, 42, 1104-1106.

MARAVITA, A., SPEnce, C., \& Driver, J. (2003). Multisensory integration and the body schema: Close to hand and within reach. Current Biology, 13, R531-R539.

Mon-Williams, M., Wann, J. P., Jenkinson, M., \& Rushton, K. (1997). Synaesthesia in the normal limb. Proceedings of the Royal Society of London: Series B, 264, 1007-1010.

Niebauer, C. L., Aselage, J., \& Schutte, C. (2002). Hemispheric interaction and consciousness: Degree of handedness predicts the intensity of a sensory illusion. Laterality, 7, 85-96.

NiELSEN, T. I. (1963). Volition: A new experimental approach. Scandinavian Journal of Psychology, 4, 225-230.

PaVAni, F., Spence, C., \& Driver, J. (2000). Visual capture of touch: Out-of-the-body experiences with rubber gloves. Psychological Science, 11, 353-359.

Peled, A., Ritsner, M., Hirschmann, S., Geva, A. B., \& Modai, I. (2000). Touch feel illusion in schizophrenic patients. Biological Psychiatry, 48, 1105-1108.

RAMAchandRan, V. S., Altschuler, E. L., \& Hillyer, S. (1997). Mirror agnosia. Proceedings of the Royal Society of London: Series B, 264, 645-647.

Ramachandran, V. S., \& Rogers-Ramachandran, D. (1996). Synaesthesia in phantom limbs induced with mirrors. Proceedings of the Royal Society of London: Series B, 263, 377-386.

RAMACHANDRAN, V. S., Rogers-RAMACHANDRAN, D., \& CobB, S. (1995). Touching the phantom limb. Nature, 377, 489-490.

Ro, T., Wallace, R., Hagedorn, J. C., Farnè, A., \& Pienkos, E. (2004). Visual enhancing of tactile perception in posterior parietal cortex. Journal of Cognitive Neuroscience, 16, 24-30.

Rossetti, Y., Desmurget, M., \& Prablanc, C. (1995). Vectorial coding of movement: Vision, proprioception, or both? Journal of Neurophysiology, 74, 457-463.

Sathian, K., Greenspan, A. I., \& Wolf, S. L. (2000). Doing it with mirrors: A case study of a novel approach to neurorehabilitation. Neurorehabilitation \& Neural Repair, 14, 73-76.

TASTEVIN, J. (1937). En partant de l'expérience d'Aristote: Les déplacements artificiels des parties du corps ne sont pas suivis par le sentiment de ces parties ni par les sensations qu'on peut y produire [Starting from Aristotle's experiment: The artificial displacements of parts of the body are not followed by feeling in these parts or by the sensations which can be produced there]. L'Encephale, 32, 57-84, 140-158.

Welch, R. B., Widawski, M. H., Harrington, J., \& WarRen, D. H. (1979). An examination of the relationship between visual capture and prism adaptation. Perception \& Psychophysics, 25, 126-132.

(Manuscript received October 15, 2003; revision accepted for publication April 22, 2004.) 